\title{
Hypoxia induces the proliferation of endothelial progenitor cells via upregulation of Apelin/APLNR/MAPK signaling
}

\author{
JINGCHANG ZHANG ${ }^{1}$, QIMING LIU ${ }^{2}$, ZHENFEI FANG $^{2}$, XINQUN HU ${ }^{2}$, \\ FENG HUANG ${ }^{3}$, LIANG TANG ${ }^{2}$ and SHENGHUA ZHOU ${ }^{2}$ \\ ${ }^{1}$ Department of Cardiology, The Third Affiliated Hospital of Guangxi Medical University, Nanning, Guangxi 530000; \\ ${ }^{2}$ Department of Cardiology, The Second Xiangya Hospital of Central South University, Changsha, Hunan 410000; \\ ${ }^{3}$ Department of Cardiology, The First Affiliated Hospital of Guangxi Medical University, Nanning, Guangxi 530000, P.R. China
}

Received September 18, 2014; Accepted June 11, 2015

DOI: $10.3892 / \mathrm{mmr} .2015 .4691$

\begin{abstract}
Endothelial progenitor cells (EPCs) can form new vessels through differentiation into endothelial cells (ECs), thus being important in the prevention of hypoxia/ischemia. Apelin can activate different signaling pathways through its receptor, APLNR, which regulate diverse biological functions, including cardiovascular function. However, the molecular mechanism by which Apelin mediates hypoxia-induced EPCs proliferation remain to be fully elucidated. The present study aimed to determine the role of Apelin/APLNR signaling in hypoxia-induced proliferation of EPCs. MTT assay was used to determine cell proliferation. Reverse transcription-quantitative polymerase chain reaction and western blotting analysis were conducted to examine mRNA and protein expression. It was revealed that hypoxia promoted the proliferation of the EPCs. Further investigation demonstrated that hypoxia promoted the expression levels of hypoxia-inducible factor (HIF)-1 $\alpha$, Apelin and APLNR in the EPCs. In addition, upregulation of Apelin or APLNR promoted the hypoxia-induced proliferation of the EPCs, while knockdown of Apelin or APLNR by small interfering RNA suppressed the hypoxia-induced proliferation of the EPCs, suggesting that the Apelin/APLNR axis is involved in hypoxia-induced proliferation of EPCs. Furthermore, pretreatment of the EPCs with SB-239063 or PD98059, two inhibitors of mitogen-activated protein kinase (MAPK), eliminated the Apelin upregulation-induced EPC proliferation, suggesting that MAPK signaling is a downstream effecter of Apelin/APLNR in EPCs. Therefore, the findings of the present study indicated that the production of HIF-1 $\alpha$, induced by hypoxia, activated the Apelin/APLNR and the downstream
\end{abstract}

Correspondence to: Professor Shenghua Zhou, Department of Cardiology, The Second Xiangya Hospital of Central South University, 139 People's Middle Road, Changsha, Hunan 410000, P.R. China

E-mail: xyshenghuazhou@163.com

Key words: endothelial progenitor cell, proliferation, Apelin, mitogen-activated protein kinase, hypoxia
MAPK signaling pathways, leading to upregulated proliferation of the EPCs. These findings suggested that Apelin/APLNR signaling may be used as a potential therapeutic target for hypoxic/ischemic injury.

\section{Introduction}

Endothelial progenitor cells (EPCs) can differentiate into endothelial cells (ECs) and are important in angiogenesis (1). It has been demonstrated that oxidative stress-induced endothelial dysfunction can cause ischemia/reperfusion injury, and the proliferation and differentiation of EPCs are essential for the treatment of this type of injury. Hypoxia has been demonstrated to mobilize EPCs from the bone marrow into the peripheral blood (2), and induce ECs to secret chemokines for the recruitment and migration of EPCs to hypoxic tissues (3). In addition, hypoxia can induce the expression of hypoxia-inducible factor- $1 \alpha(\mathrm{HIF}-1 \alpha)$, an important transcriptional factor that is involved in the proliferation and differentiation of EPCs (4).

Apelin is an endogenous ligand for the $G$ protein-coupled receptor, APLNR (5). It has been suggested that Apelin/APLNR signaling can be activated by HIF-1 $\alpha$ in hypoxia neonatal cardiomyocytes (6). In addition, downregulated serum levels of Apelin are associated with EPC mobilization induced by acute myocardial infarction (7) and Apelin deficiency impairs EPC sprouting in ischemia-reperfusion injury (8). These findings suggest that Apelin may be involved in the regulation of EPC mobilization and sprouting. However, whether the Apelin/APLNR signaling is involved in hypoxia-induced EPC proliferation has not been investigated previously.

The mitogen-activated protein kinase (MAPK) signaling pathway is important in the regulation of cellular survival, proliferation, differentiation and migration (9). It has been demonstrated that the MAPK signaling pathway is involved in the regulation of EPC proliferation, for example, the effect of granulocyte macrophage colony-stimulating factor on the modulation of EPC proliferation is mediated by the MAPK signaling pathway (10). Our previous study revealed that Apelin/APLNR signaling promotes hypoxia-induced proliferation of EPCs via phosphoinositide 3 kinase/Akt signaling (11). However, whether Apelin/APLNR signaling regulates EPC proliferation via MAPK signaling remains to be elucidated 
The aim of the present study was to determine the underlying molecular mechanisms by which Apelin/APLNR signaling regulates the hypoxia-induced proliferation of EPCs.

\section{Materials and methods}

Cell isolation and culture. EPCs were isolated from the umbilical cord at the Second Xiangya Hospital of Central South University in September 2012. Briefly, umbilical cord tissue sample was diluted with Dulbecco's phosphate-buffered saline at a ratio of $1: 1$ and overlaid onto $1.077 \mathrm{~g} / \mathrm{ml}$ Ficoll-Paque $^{\mathrm{TM}}$ Premium (GE Healthcare Bio-Sciences, Pittsburgh, PA, USA) prior to centrifugation for $30 \mathrm{~min}$ at $400 \mathrm{x} \mathrm{g}$. The monocytes were collected and seeded into tissue culture plates coated with fibronectin (EMD Millipore, Billerica, MA, USA), and cultured in endothelial growth medium (EGM)-2 (Thermo Fisher Scientific, Waltham, MA, USA) supplemented with $20 \%$ fetal bovine serum (FBS; Tianhang Biological Technology Co., Ltd., Hangzhou, China) at $37^{\circ} \mathrm{C}$ in a humidified incubator containing $5 \% \mathrm{CO}_{2}$. The culture media was changed every two days until EPC colonies appeared. Typical colonies appear between days 5 and 10, and were passaged at sub-confluence. The present study was approved by the ethics committee of the Second Xiangya Hospital of Central South University (Changsha, China).

Hypoxia treatment of the EPCs. Prior to hypoxia treatment, the EPCs $\left(5 \times 10^{5}\right)$ were cultured in serum-free EGM-2 at $37^{\circ} \mathrm{C}$, $5 \% \mathrm{CO}_{2}$ for $12 \mathrm{~h}$. Subsequently, the EPCs were cultured in EGM-2 containing $20 \% \mathrm{FBS}$ at $37,1 \% \mathrm{O}_{2} / 94 \% \mathrm{~N}_{2} / 5 \% \mathrm{CO}_{2}$ for $6,12,24$ and $48 \mathrm{~h}$.

Cell proliferation assay. An MTT assay was performed to determine the cell proliferation. In brief, $10 \mathrm{mg} / \mathrm{ml}$ MTT (Thermo Fisher Scientific) was added to the medium following culture. Following incubation for $4 \mathrm{~h}$, the reaction was terminated by removal of the supernatant and the addition of $100 \mu \mathrm{l}$ dimethyl sulfoxide (DMSO; Thermo Fisher Scientific) to dissolve the formazan product. After $30 \mathrm{~min}$, the optical density (OD) of each well was measured at $570 \mathrm{~nm}$ using a microplate reader (ELx808; Bio-Tek Instruments, City, ST, USA).

Reverse transcription-quantitative polymerase chain reaction (RT-qPCR) analysis. TRIzol reagent (Life Technologies, Carlsbad, CA, USA) was used to extract the total RNA from the EPCs, according to the manufacturer's instructions. The total RNA was reverse transcribed into cDNA using a RevertAid First-Strand cDNA Synthesis kit (Thermo Fisher Scientific), according to the manufacturer's instructions. The mRNA expression level was determined using iQTM SYBR Green Supermix (Bio-Rad Laboratories, Inc., Hercules, CA, USA), according to the manufacture's instructions. The reaction mixture contained $1 \mu \mathrm{l}$ cDNA solution, $10 \mu \mathrm{l}$ PCR master mix, $2 \mu \mathrm{l}$ primers (Sangon Biotech Co., Ltd., Shanghai, China), and $7 \mu 1 \mathrm{H}_{2} \mathrm{O}$ were mixed to obtain a final reaction volume of $20 \mu \mathrm{l}$. The qPCR was conducted using an ABI7500 PCR system (Invitrogen; Thermo Fisher Scientific, Inc.) with the following reaction conditions: Pre-degeneration at $95^{\circ} \mathrm{C}$ for $1 \mathrm{~min}$, followed by 40 cycles of $95^{\circ} \mathrm{C}$ for $15 \mathrm{sec}, 60^{\circ} \mathrm{C}$ for $15 \mathrm{sec}$ and $72^{\circ} \mathrm{C}$ for $1 \mathrm{~min}$. The specific primer pairs used were as follows: HIF-1 $\alpha$, sense 5'-GAACGTCGAAAAGAAAAGTCTCG-3' and antisense 5'-CCTTATCAAGATGCGAACTCACA-3'; Apelin, sense 5'-GTCTCCTCCATAGATTGGTCTGC-3' and antisense 5'-GGAATCATCCAAACTACAGCCAG-3'; APLNR, sense 5'-CTCTGGACCGTGTTTCGGAG-3' and antisense 5'-GGTACGTGTAGGTAGCCCACA-3'; and GAPDH (as an internal reference) sense 5'-GGAGCGAGATCCCTCCAAAAT-3' and antisense 5'-GGCTGTTGTCATACTTCTCATGG-3'. Independent experiments were repeated three times. The relative expression levels of mRNA were analyzed using the $2^{-\Delta \Delta \mathrm{Ct}}$ method (12).

Transfection. Transfection of the cells was performed using Lipofectamine 2000 (Invitrogen; Thermo Fisher Scientific, Inc.), according to the manufacturer's instructions. For functional analysis, the EPCs were transfected with either an Apelin plasmid, Apelin-specific siRNA, an APLNR plasmid or APLNR-specific siRNA (Nlunbio, Changsha, China).

Western blot analysis. The cells were solubilized in cold radioimmunoprecipitation assay lysis buffer. The proteins were quantified using a Bicinchoninic Acid Protein Assay kit (Thermo Fisher Scientific, Inc.), according to the manufacturer's instructions. The resulting proteins $(50 \mu \mathrm{g})$ were separated with 12\% SDS-PAGE (Beyotime Institute of Biotechnology, Shanghai, China) and transferred onto a polyvinylidene difluoride (PVDF) membrane (Thermo Fisher Scientific), which was then incubated with Tris-buffered saline with $1 \%$ Tween 20 (Thermo Fisher Scientific) containing 5\% skimmed milk at $4^{\circ} \mathrm{C}$ for overnight. Following incubation, the PVDF membrane was incubated with mouse anti-HIF- $\alpha$, mouse anti-Apelin, mouse anti-APLNR and mouse anti-GAPDH primary antibodies (Abcam, Cambridge, UK), respectively, at room temperature for $3 \mathrm{~h}$. Following washing with PBS with 1\% Tween 20 (PBST; Thermo Fisher Scientific) three times, the PVDF membrane was incubated with rabbit anti-mouse secondary antibody (Abcam) at room temperature for $1 \mathrm{~h}$. Following washing with PBST three times, an ECL kit (Pierce Chemical, Rockford, IL, USA) was used to perform chemiluminescent detection. The relative expression of proteins were determined using Image-Pro plus software 6.0, presented as the density ratio, compared with GAPDH.

Statistical analysis. All data are expressed as the mean \pm standard deviation of three independent experiments. Statistical analyses of differences were performed using one-way analysis of variance with SPSS 17 software (SPSS, Inc., Chicago, IL, USA). $\mathrm{P}<0.05$ was considered to indicate a statistically significant difference.

\section{Results}

Hypoxia promotes EPC proliferation. The proliferation rates of the EPCs were examined under hypoxia and normoxia using an MTT assay. As shown in Fig. 1, following culture in hypoxia for $6,12,24$, and $48 \mathrm{~h}$, the relative proliferation rates of the EPCs were notably upregulated, compared with the EPCs cultured in normoxia, indicating that hypoxia promoted EPC proliferation. 


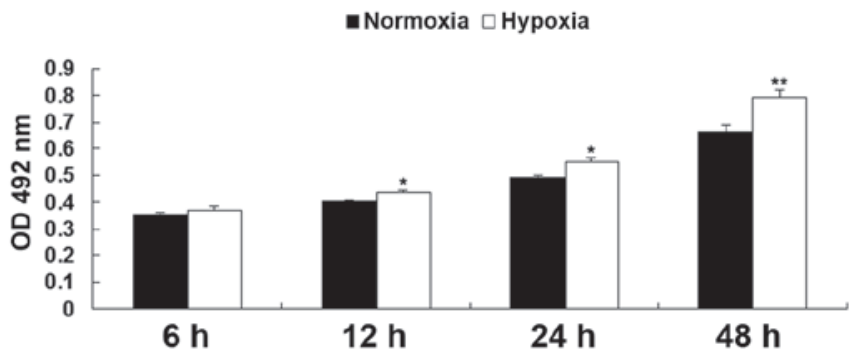

Figure 1. An MTT assay was performed to determine the cell proliferation of endothelial progenitor cells cultured under normoxia or hypoxia for $6 \mathrm{~h}$, $12 \mathrm{~h}, 24 \mathrm{~h}$ and $48 \mathrm{~h}$. ${ }^{*} \mathrm{P}<0.05$, vs. normoxia; ${ }^{* *} \mathrm{P}<0.01$, vs. normoxia. Data are expressed as the mean \pm standard deviation. OD, optical density.

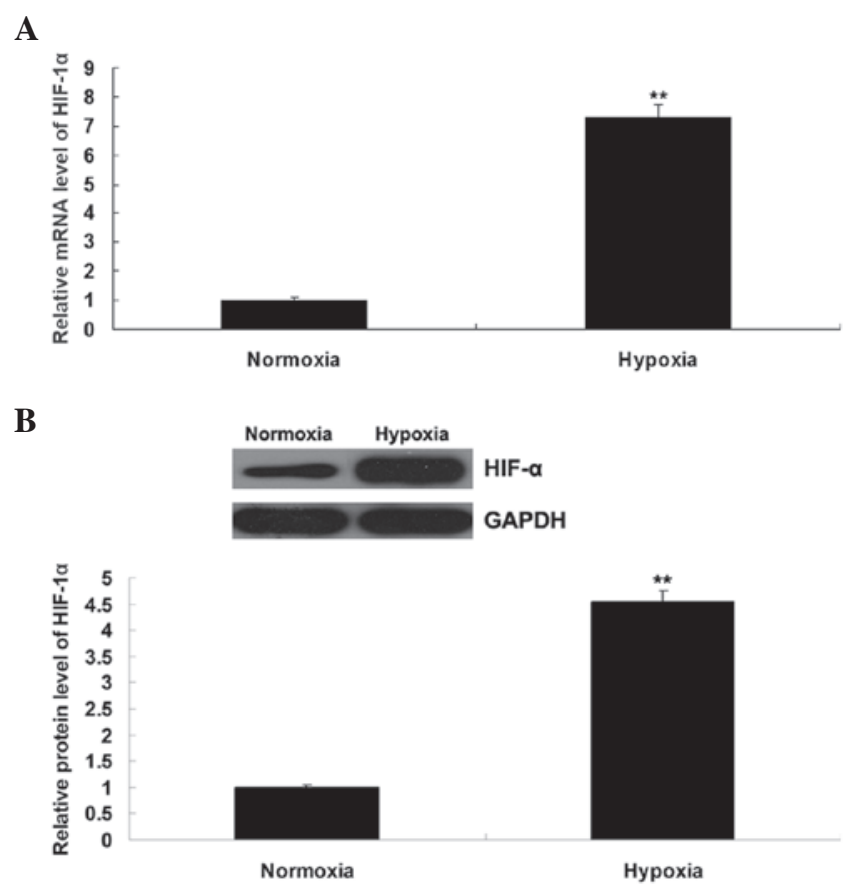

Figure 2. (A) Reverse transcription-quantitative polymerase chain reaction was performed to examine the mRNA expression of HIF-1 $\alpha$ in EPCs cultured under normoxia or hypoxia. GAPDH was used as an internal reference. ${ }^{* *} \mathrm{P}<0.01$, vs. normoxia. (B) Western blotting was performed to examine the protein expression of HIF-1 $\alpha$ in EPCs cultured under normoxia or hypoxia. GAPDH was used as an internal reference. ${ }^{* *} \mathrm{P}<0.01$ vs. normoxia. Data are expressed as the mean \pm standard deviation. HIF $1 \alpha$, hypoxia-inducible factor-1 $\alpha$; EPC, endothelial progenitor cell.

Hypoxia induces the upregulation of HIF-l $\alpha$ and Apelin/APLNR signaling. The expression level of HIF-1 $\alpha$ was further determined by performing RT-qPCR and western blot assays. As shown in Fig. 2A and B, the mRNA and protein expression levels of HIF-1 $\alpha$ were notably upregulated in the EPCs cultured under hypoxia, compared with those cultured in normoxia. As Apelin/APLNR signaling has been found to be regulated by HIF-1 $\alpha$, the present study further determined the expression levels of Apelin and APLNR in EPCs cultured under hypoxia or normoxia, respectively. As shown in Fig. 3A and $\mathrm{B}$, the mRNA and protein expression levels of Apelin and APLNR were significantly upregulated in the EPCs cultured under hypoxia, compared with those cultured in normoxia. In addition, the secretion of Apelin was determined using ELISA. As shown in Fig. 3C, the secretion level of Apelin was also
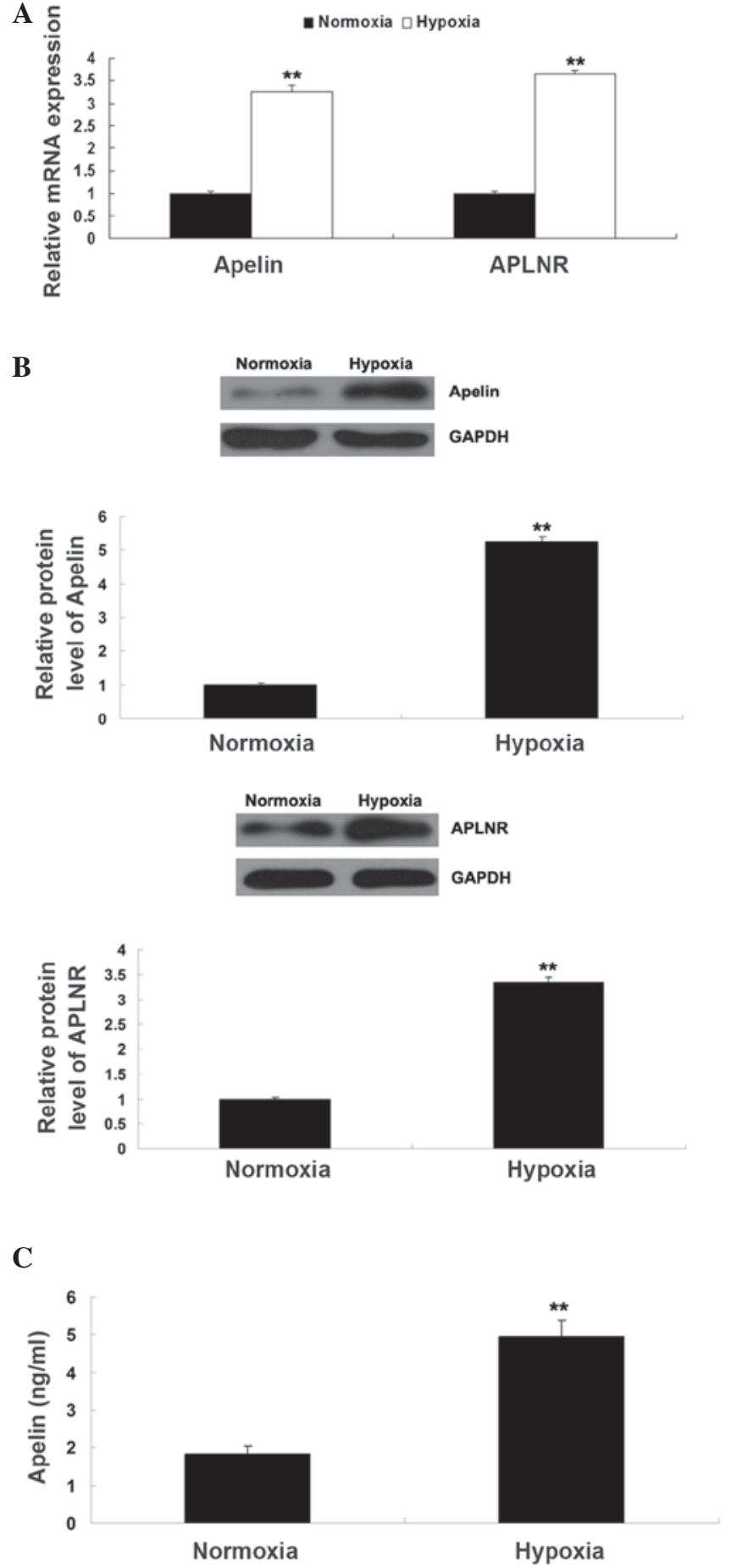

Figure 3. (A) Reverse transcription-quantitative polymerase chain reaction was performed to examine the mRNA expression levels of Apelin and APLNR in EPCs cultured under normoxia or hypoxia. GAPDH was used as an internal reference. ${ }^{* *} \mathrm{P}<0.01$, vs. normoxia. (B) Western blotting was performed to examine the protein expression levels of Apelin and APLNR in EPCs cultured under normoxia or hypoxia. GAPDH was used as an internal reference. ${ }^{* *} \mathrm{P}<0.01$, vs. normoxia. (C) ELISA assay was performed to examine the secretion level of Apelin in EPCs cultured under normoxia or hypoxia. ${ }^{* *} \mathrm{P}<0.01$, vs. normoxia. Data are expressed as the mean \pm standard deviation. EPC, endothelial progenitor cell; APLNR, Apelin receptor.

increased in the EPCs cultured under hypoxia, compared with those cultured in normoxia. Taken together, these findings suggested that hypoxia induced the upregulation of HIF-1 $\alpha$ and its downstream Apelin/APLNR signaling in the EPCs. 
A

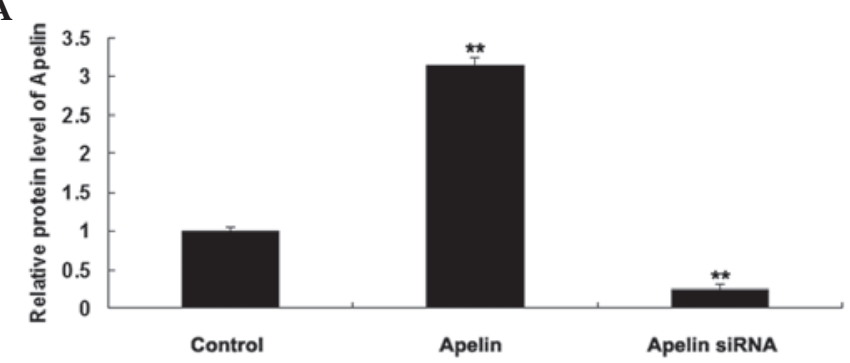

B
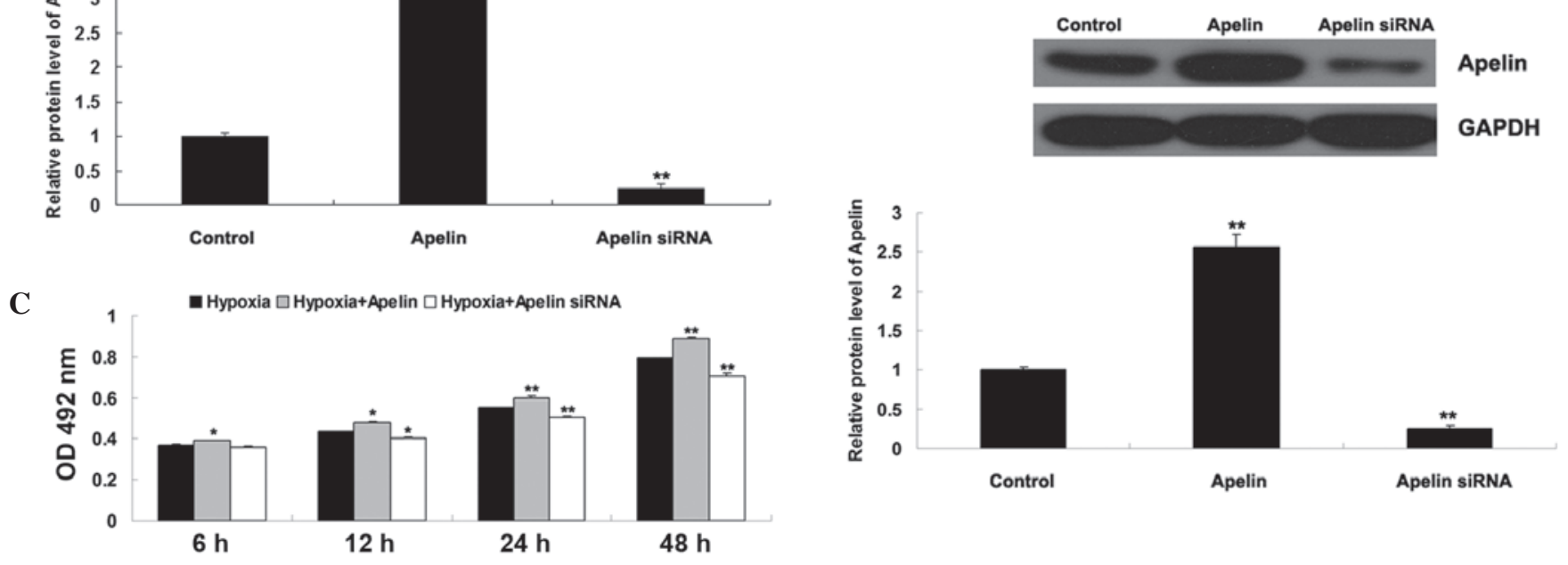

Figure 4. (A) Reverse transcription-quantitative polymerase chain reaction was performed to examine the mRNA expression of Apelin in EPCs transfected with either the Apelin plasmid or Apelin siRNA. GAPDH was used as an internal reference. Untransfected cells were used as a control. ${ }^{* *}$ P $<0.01$, vs. control. (B) Western blotting was performed to examine the protein expression of Apelin in EPCs transfected with either the Apelin plasmid or Apelin siRNA. GAPDH was used as an internal reference. ${ }^{* *} \mathrm{P}<0.01$, vs. control. (C) MTT assay was performed to determine the cell proliferation rates of EPCs in each group at 6 h, 12 h, 24 h and 48 h. Hypoxia: EPCs cultured under hypoxia. Hypoxia + Apelin: EPCs transfected with the Apelin plasmid and cultured under hypoxia. Hypoxia + Apelin siRNA: EPCs transfected with Apelin siRNA and cultured under hypoxia. ${ }^{*} \mathrm{P}<0.05$, vs. hypoxia. ${ }^{* *} \mathrm{P}<0.01$, vs. hypoxia. Data are expressed as the mean \pm standard deviation. EPC, endothelial progenitor cell; APLNR, Apelin receptor; OD, optical density; siRNA, small interfering RNA.

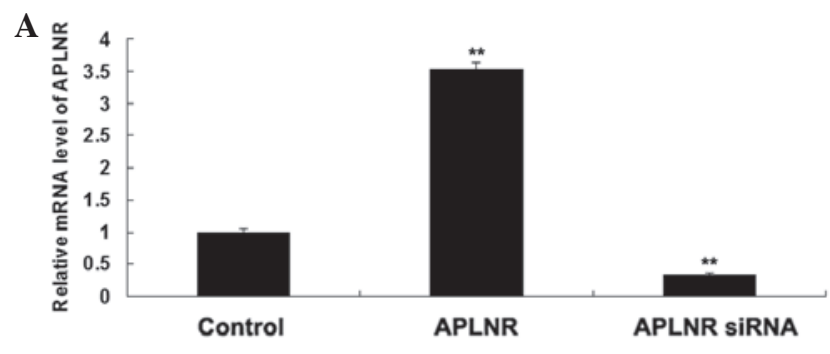

B
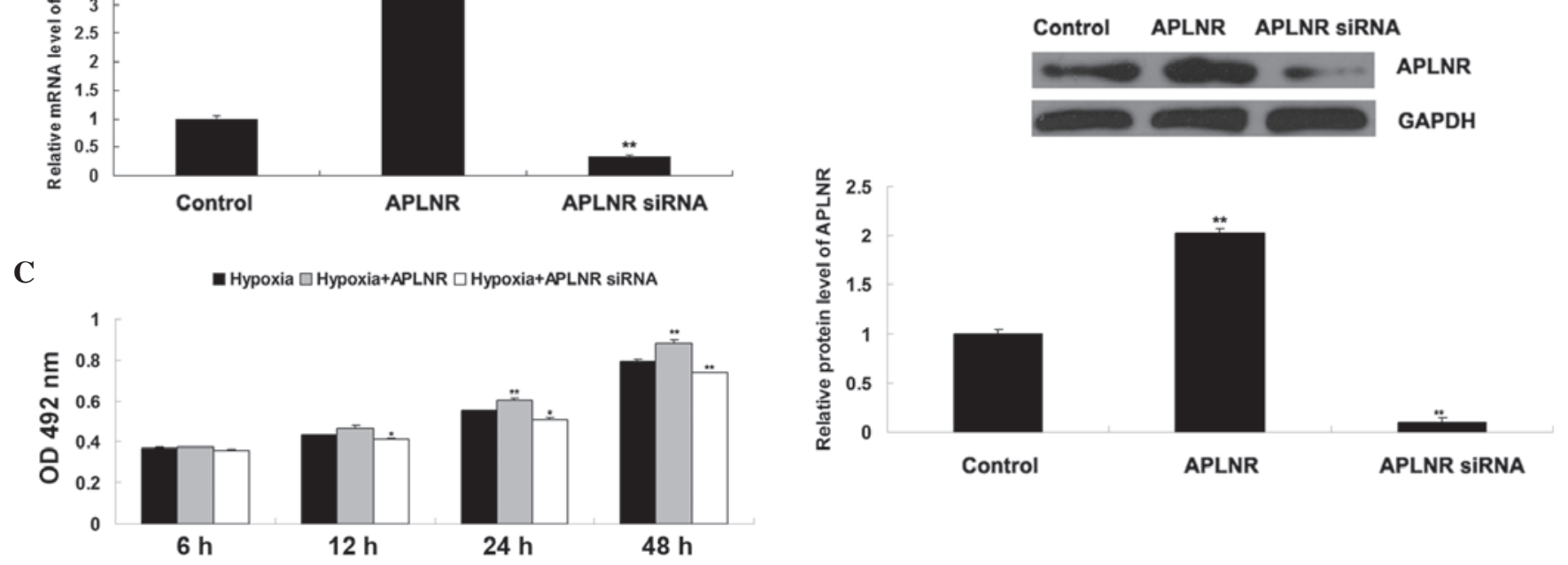

Figure 5. (A) Reverse transcription-quantitative polymerase chain reaction was performed to examine the mRNA expression of APLNR in EPCs transfected with either tha APLNR plasmid or APLNR siRNA. GAPDH was used as an internal reference. Untransfected cells were used as a control. ${ }^{* *} \mathrm{P}<0.01$ vs. control. (B) Western blotting was performed to examine the protein expression of APLNR in EPCs transfected with either the APLNR plasmid or APLNR siRNA, GAPDH was used as an internal reference. Untransfected cells were used as a control. ${ }^{* *} \mathrm{P}<0.01$, vs. control. (C) MTT assay was performed to determine the cell proliferation of EPCs in each group at 6 h, 12 h, 24 h and 48 h. Hypoxia, EPCs cultured under hypoxia; Hypoxia + APLNR, EPCs transfected with APLNR plasmid and cultured under hypoxia; Hypoxia + APLNR siRNA, EPCs transfected with APLNR siRNA and cultured under hypoxia. *P<0.05, vs. hypoxia. ${ }^{* *} \mathrm{P}<0.01$, vs. hypoxia. Data are expressed as the mean \pm standard deviation. EPC, endothelial progenitor cell; APLNR, Apelin receptor; OD. optical density; siRNA, small interfering RNA.

Role of Apelin/APLNR signaling in EPC proliferation. The role of Apelin/APLNR signaling was further investigated in the proliferation of EPCs cultured under hypoxia or normoxia, respectively. The EPCs were initially transfected either with an Apelin plasmid or siRNA, in order to upregulate or downregulate Apelin/APLNR signaling, respectively. As shown in Fig. 4A and B, the mRNA and protein expression levels of Apelin increased in the EPCs transfected with the Apelin plasmid, and reduced in the EPCs transfected with the Apelin siRNA. The present study subsequently determined the proliferation of EPCs cultured under hypoxia or normoxia using an MTT assay. As shown in Fig. 4C, the proliferation of the EPCs in normoxia + Apelin group was higher than that in the normoxia group, suggesting that the upregulation of 
Apelin enhanced EPC proliferation. In addition, the proliferation of the EPCs in the hypoxia + Apelin siRNA group was lower than that in the hypoxia group, suggesting that the inhibition of Apelin suppressed hypoxia-induced EPC proliferation.

To further confirm the hypothesis that Apelin/APLNR signaling is involved in EPC proliferation, the present study transfected EPCs with either an APLNR plasmid or siRNA. As shown in Fig.5A and 5B, the mRNA and protein expression levels of APLNR were increased in the EPCs transfected with the APLNR plasmid, but reduced in the EPCs transfected with APLNR siRNA. The proliferation of EPCs cultured under hypoxia or normoxia was then determined using an MTT assay. As shown in Fig.5C, the proliferation of EPCs in the normoxia + APLNR group was higher than that in the normoxia group, suggesting that upregulation of APLNR enhanced EPC proliferation. In addition, the proliferation of EPCs in the hypoxia + APLNR siRNA group was lower than that in the hypoxia group, suggesting that the inhibition of APLNR suppressed hypoxia-induced EPC proliferation. Taken together, these findings suggested that Apelin/APLNR signaling is a key regulator of hypoxia-induced EPC proliferation.

MAPK signaling pathway is a downstream effecter of Apelin/APLNR signaling during hypoxia-induced EPC proliferation. As the MAPK signaling pathway has been suggested to be involved in hypoxia-induced EPC proliferation, the present study further investigated whether the MAPK signaling pathway is a downstream effecter of Apelin/APLNR signaling during hypoxia-induced EPC proliferation. The P38 MAPK inhibitor, SB-239063, or ERK MAPK inhibitor, PD98059, was used to treat EPCs, which had been transfected with the Apelin plasmid. A total of four groups of EPCs were cultured under hypoxia, as follows: Control group without any treatment, Apelin group, Apelin + SB-239063 group, and Apelin + PD98059 group. As shown in Fig. 6, the upregulation of Apelin promoted EPC proliferation under hypoxia, which was abrogated by pretreatment with SB-239063 or PD98059, respectively. These findings suggested that inhibition of MAPK signaling suppressed hypoxia-induced EPC proliferation. Taken together, it was suggested that MAPK pathway is a downstream effecter of Apelin/APLNR signaling during hypoxia-induced EPC proliferation.

\section{Discussion}

The Apelin/APLNR signaling pathway has been demonstrated to be closely associated with cardiovascular functions in the systemic circulation (13). In the present study, hypoxia-induced upregulation of HIF- $\alpha$ and Apelin/APLNR signaling was found to be involved in the proliferation of EPCs. In addition, the results suggested that MAPK signaling is involved in Apelin/APLNR-mediated EPC proliferation.

Hypoxia has been demonstrated to be involved in the regulation of cellular processes of EPCs (14). In the present study, hypoxia notably enhanced the proliferation of EPCs. Lee et al reported that hypoxia inhibits senescence of EPCs in aged humans (15). In addition, hypoxia/ischemia triggers EPCs to proceed from bone marrow into the peripheral blood, and

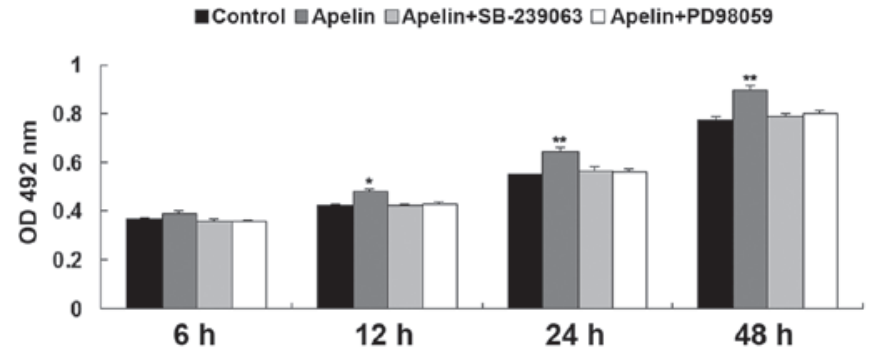

Figure 6. MTT assay was performed to determine the cell proliferation of EPCs in each group at 6 h, 12 h, $24 \mathrm{~h}$ and 48 h. Control, EPCs cultured under hypoxia; Apelin, EPCs cultured under hypoxia; Apelin + SB-239063, EPCs transfected with the Apelin plasmid were treated with SB-239063 and cultured under hypoxia. Apelin + PD98059, EPCs transfected with the Apelin plasmid were treated with PD98059 and cultured under hypoxia. ${ }^{*} \mathrm{P}<0.05$ vs control; ${ }^{* *} \mathrm{P}<0.01$, vs. control. Data are expressed as the mean \pm standard deviation. EPC, endothelial progenitor cell; APLNR, Apelin receptor; OD, optical density.

EPCs can differentiate into ECs following migration into the site of hypoxia/ischemia in tissues (16). In addition, HIF-1 $\alpha$ pis important in the regulation of angiogenesis. Overexpression of HIF-1 $\alpha$ can promote the differentiation of EPCs into ECs, while inhibition of the expression of HIF-1 $\alpha$ suppresses the differentiation of EPCs into ECs, as well as the expression of vascular endothelial growth factor (VEGF), VEGF receptor 2, eNOS, and the production of NO $(4,17,18)$. The present study demonstrated that the expression level of HIF- $\alpha$ was notably upregulated in EPCs cultured under hypoxia.

Apelin/APLNR signaling is involved in various physiological and pathological functions in the cardiovascular system. Apelin/APLNR signaling promotes angiogenesis and improves cardiac functional recovery post-myocardial infarction (19). In addition, Lv et al observed that Apelin/APLNR signaling regulates the proliferation of vascular smooth muscle cells (20), and Kasai et al found that inhibition of Apelin/APLNR signaling inhibits the proliferation of ECs (21). In the present study, the expression levels of Apelin and APLNR were found to be increased in EPCs cultured under hypoxia. The Apelin/APLNR signaling is also involved in the mobilization of EPCs following acute myocardial infarction (7). The present study hypothesized that the increased expression of Apelin/APLNR is associated with the upregulation of HIF- $\alpha$ in hypoxia-treated EPCs, as the association between HIF-1 $\alpha$ and Apelin/APLNR signaling has been reported in other cell types. Glassford et al demonstrated that HIF-1 $\alpha$ regulated the expression of Apelin in hypoxia-treated adipocytes (22). Ronkainen et al found that the upregulation of Apelin was eliminated by HIF inhibitory PAS protein in cardiomyocytes cultured under hypoxia (23). These findings suggest that the Apelin/APLNR signaling is mediated by the HIF pathway in adipocytes and cardiomyocytes cultured under hypoxia. In addition, the present study further demonstrated that knockdown of Apelin/APLNR signaling significantly inhibited hypoxia-induced EPC proliferation. These findings are the first time, to the best of our knowledge, to suggest that Apelin/APLNR signaling is involved in regulating the proliferation of EPCs cultured under hypoxia.

The underlying mechanisms by which Apelin/APLNR signaling regulates hypoxia-induced EPCs proliferation 
requires further investigation. The present study demonstrated that inhibition of the p38 and ERK MAPK signaling pathways suppressed hypoxia-induced proliferation of EPCs transfected with an Apelin plasmid. Similar findings have been observed in other cell types. Apelin/APLNR suppresses the production and release of reactive oxygen species and promotes the expression of anti-oxidant enzymes via the MAPK signaling pathway in adipocytes (24). Yang et al revealed that Apelin/APLNR signaling protects the brain against ischemia/reperfusion injury via activation of the ERK MAPK signaling pathway (25). However, whether the MAPK signaling pathway is involved in Apelin/APLNR signaling-mediated EPC proliferation remains to be elucidated. The data of the present study suggested, for the first time that the p38 and ERK MAPK signaling pathway acts as a downstream effecter of Apelin/APLNR signaling during hypoxia-induced EPC proliferation.

In conclusion, the results of the present study suggested that hypoxia-induced upregulation of HIF-1 $\alpha$ activates Apelin/APLNR signaling, which promotes the proliferation of EPCs via the downstream MAPK signaling pathway. These findings suggested that the Apelin/APLNR signaling may be applied as a therapeutic target for hypoxic/ischemic injury.

\section{References}

1. Endemann DH and Schiffrin EL: Endothelial dysfunction. J Am Soc Nephrol 15: 1983-1992, 2004.

2. Schröder K, Kohnen A, Aicher A, Liehn EA, Büchse T, Stein S, Weber C, Dimmeler S and Brandes RP: NADPH oxidase Nox2 is required for hypoxia-induced mobilization of endothelial progenitor cells. Circ Res 105: 537-544, 2009.

3. Simons D, Grieb G, Hristov M, Pallua N, Weber C, Bernhagen J and Steffens G: Hypoxia-induced endothelial secretion of macrophage migration inhibitory factor and role in endothelial progenitor cell recruitment. J Cell Mol Med 15: 668-678, 2011.

4. Jiang M, Wang CQ, Wang BY and Huang DJ: Inhibitory effect of siRNA targeting HIF-lalpha on differentiation of peripheral blood endothelial progenitor cells. Ai Zheng 24: 1293-1300, 2005 (In Chinese).

5. Kleinz MJ and Baxter GF: Apelin reduces myocardial reperfusion injury independently of PI3K/Akt and P70S6 kinase. Regul Pept 146: 271-277, 2008.

6. Zhang Z, Yu B and Tao GZ: Apelin protects against cardiomyocyte apoptosis induced by glucose deprivation. Chin Med J (Engl) 122: 2360-2365, 2009.

7. Ye J, Ni P, Kang L and Xu B: Apelin and vascular endothelial growth factor are associated with mobilization of endothelial progenitor cells after acute myocardial infarction. J Biomed Res 26: 400-409, 2012.

8. Wang W, McKinnie SM, Patel VB, Haddad G, Wang Z, Zhabyeyev P, Das SK, Basu R, McLean B, Kandalam V, et al: Loss of Apelin exacerbates myocardial infarction adverse remodeling and ischemia-reperfusion injury: Therapeutic potential of synthetic Apelin analogues. J Am Heart Assoc 2: e000249, 2013.
9. Kyriakis JM and Avruch J: Mammalian MAPK signal transduction pathways activated by stress and inflammation: A 10-year update. Physiol Rev 92: 689-737, 2012.

10. Qiu C, Xie Q, Zhang D, Chen Q, Hu J and Xu L: GM-CSF induces cyclin D1 expression and proliferation of endothelial progenitor cells via PI3K and MAPK signaling. Cell Physiol Biochem 33: 784-795, 2014.

11. Zhang J, Liu Q, Hu X, Fang Z, Huang F, Tang L and Zhou S: Apelin/APJ signaling promotes hypoxia-induced proliferation of endothelial progenitor cells via phosphoinositide-3 kinase/Akt signaling. Mol Med Rep 12: 3829-3834, 2015.

12. Zhong Y, Lin Y, Shen S, Zhou Y, Mao F, Guan J and Sun Q: Expression of ALDH1 in breast invasive ductal carcinoma: An independent predictor of early tumor relapse. Cancer Cell Int 13: 60, 2013.

13. O'Carroll AM, Lolait SJ, Harris LE and Pope GR: The Apelin receptor APJ: Journey from an orphan to a multifaceted regulator of homeostasis. J Endocrinol 219: R13-R35, 2013.

14. Dai T, Zheng $\mathrm{H}$ and Fu GS: Hypoxia confers protection against apoptosis via the PI3K/Akt pathway in endothelial progenitor cells. Acta Pharmacol Sin 29: 1425-1431, 2008.

15. Lee SH, Lee JH, Yoo SY, Hur J, Kim HS and Kwon SM: Hypoxia inhibits cellular senescence to restore the therapeutic potential of old human endothelial progenitor cells via the hypoxia-inducible factor-1alpha-TWIST-p21 axis. Arterioscler Thromb Vasc Biol 33: 2407-2414, 2013.

16. Machalińska A: The role of circulating endothelial progenitor cells in the development of vascular retinal diseases. Klin Oczna 115: 158-162, 2013 (In Polish).

17. Jiang M, Wang B, Wang C, He B, Fan H, Guo TB, Shao Q, Gao L and Liu Y: Inhibition of hypoxia-inducible factor-1alpha and endothelial progenitor cell differentiation by adenoviral transfer of small interfering RNA in vitro. J Vasc Res 43: 511-521, 2006.

18. Jiang M, Wang CQ, Wang BY, He B, Shao Q and Huang DJ: Overexpression of hypoxia inducible factor-1alpha (HIF-1alpha) promotes the differentiation of endothelial progenitor cell ex vivo. Zhongguo Shi Yan Xue Ye Xue Za Zhi 14: 565-570, 2006 (In Chinese).

19. Li L, Zeng H and Chen JX: Apelin-13 increases myocardial progenitor cells and improves repair postmyocardial infarction. Am J Physiol Heart Circ Physiol 303: H605-H618, 2012.

20. Lv D, Li H and Chen L: Apelin and APJ, a novel critical factor and therapeutic target for atherosclerosis. Acta Biochim Biophys Sin (Shanghai) 45: 527-533, 2013.

21. Kasai A, Ishimaru Y, Higashino K, Kobayashi K, Yamamuro A, Yoshioka Y and Maeda S: Inhibition of Apelin expression switches endothelial cells from proliferative to mature state in pathological retinal angiogenesis. Angiogenesis 16: 723-734, 2013.

22. Glassford AJ, Yue P, Sheikh AY, Chun HJ, Zarafshar S, Chan DA, Reaven GM, Quertermous T and Tsao PS: HIF-1 regulates hypoxia- and insulin-induced expression of Apelin in adipocytes. Am J Physiol Endocrinol Metab 293: E1590-E1596, 2007.

23. Ronkainen VP, Ronkainen JJ, Hänninen SL, Leskinen H, Ruas JL, Pereira T, Poellinger L, Vuolteenaho O and Tavi P: Hypoxia inducible factor regulates the cardiac expression and secretion of Apelin. FASEB J 21: 1821-1830, 2007.

24. Than A, Zhang X, Leow MK, Poh CL, Chong SK and Chen P: Apelin attenuates oxidative stress in human adipocytes. J Biol Chem 289: 3763-3774, 2014.

25. Yang Y, Zhang X, Cui H, Zhang C, Zhu C and Li L: Apelin-13 protects the brain against ischemia/reperfusion injury through activating PI3K/Akt and ERK1/2 signaling pathways. Neurosci Lett 568: 44-49, 2014. 